\title{
Converging on a new role for analogy in problem solving and retrieval: When two problems are better than one
}

\author{
KENNETH J. KuRTZ \\ Binghamton University, Binghamton, New York \\ AND \\ JEFFREY LOEWENSTEIN \\ University of Texas, Austin, Texas
}

\begin{abstract}
People often fail to retrieve examples analogous to a current problem or situation. There is good evidence that comparing structurally matching cases facilitates subsequent analogical access. However, current approaches offer little at the time of memory search to promote retrieval of a routinely encoded analogous source. We adapted Gick and Holyoak's $(1980,1983)$ classic paradigm to investigate whether comparing two unsolved problems at test promotes retrieval of a single previously studied analogue. In Experiment 1, comparison of test problems facilitated analogical problem solving. Experiment 2 showed that comparison is the critical factor since solving two test problems separately proved ineffective. In Experiment 3, comparing two problems led to greater success for participants who read a prior analogous story than those who did not, demonstrating specifically that comparison facilitates retrieval. The three studies show that analogical access is powerfully determined by problem encoding. Implications for psychological theory and real-world applications are discussed.
\end{abstract}

People often solve problems by recalling similar examples. However, many such remindings are superficially similar, based only on isolated matches at the object or feature level. If a retrieved example does not have the same structure as the target problem, it is unlikely to provide useful inferences about possible solutions. Hence, a major research goal is to explain when and why people experience analogical remindings on the basis of common relational structure, not surface similarities (Gentner, 1983, 1989). Spontaneous transfer of useful knowledge across domains is a powerful cognitive tool, so it is important to understand what makes it work and what can make it work more widely.

We know that memory retrieval is a key bottleneck because people frequently fail, especially when they lack domain expertise, to spontaneously retrieve analogous examples that they demonstrably know (Catrambone, 2002; Gentner, Rattermann, \& Forbus, 1993; Gick \& Holyoak, 1980; Holyoak \& Koh, 1987; Novick, 1988; Ross, 1989). Considerable effort has been expended on explaining similarity-based retrieval (Dunbar, 2001; Forbus, Gentner, \& Law, 1995; Hummel \& Holyoak, 1997) and exploring techniques to improve people's ability to retrieve analogies through better initial learning (Barnett \& Ceci, 2002; Goldstone \& Sakamoto, 2003; Reeves \& Weisberg, 1994). The half-helpful prescription from this prior research is
"You should have learned it right the first time." We are shifting attention to an underexplored aspect of analogical retrieval - the role of problem representation (see also Gentner, Loewenstein, \& Thompson, 2004). We provide evidence that analogical retrieval can be facilitated for stored examples that were learned in an ordinary fashion.

Prior research tells us that improving the encoding of study materials promotes subsequent analogical access. The canonical finding is that comparing structurally consistent cases greatly facilitates later spontaneous analogical retrieval of those cases to solve new problems (Catrambone \& Holyoak, 1989; Gick \& Holyoak, 1983; Loewenstein, Thompson, \& Gentner, 1999; Ross \& Kennedy, 1990). The accepted explanation is that comparing two stories fosters schema abstraction-generalization of the relational structure common to the stories - and that schema abstraction in turn yields an advantage for analogical retrieval and problem solving (Cummins Dellarosa, 1992; Gentner \& Medina, 1998; Gick \& Holyoak, 1983; Medin \& Ross, 1989). The main evidence in support of schema abstraction is that comparing initial cases improves people's ability to articulate the relations or principles shared by the stories, and that such articulation is associated with successful transfer (Gick \& Holyoak, 1983; Loewenstein et al., 1999). Thus, if people are led to represent initial stories by focusing on their structure, later

K. J. Kurtz, kkurtz@binghamton.edu 
they seem able to retrieve those schematic representations to solve analogous problems.

Schemas are thought to provide a better basis for analogical retrieval than are individual examples, because they reduce irrelevant information and enhance the encoding of relational information (Forbus et al., 1995; Hummel \& Holyoak, 1997). Representations of single examples necessarily include their specifics, whereas schemas filter out whatever is not common to all examples. Therefore, surface mismatches do not obscure a good structural match or prevent an analogue from competing successfully against superficially matching rivals. In addition, the comparison process of structural alignment highlights relational content (Clement \& Gentner, 1991; Gentner \& Medina, 1998), so a schema abstracted via comparison may encode relations more effectively than in the individual case. Rerepresentation has been posited to foster alignment of nonidentical relational predicates (Gentner \& Rattermann, 1991; Kurtz, 2005). The encoding that results, being less tied to role fillers and idiosyncrasies of setting, should transfer more easily. Consistent with this theoretical claim, people are better able to retrieve analogical matches if the examples are written using generic relational words rather than concrete, domain-specific terms (Clement, Mawby, \& Giles, 1994).

Prior research (e.g., Gick \& Holyoak, 1983; Loewenstein et al., 1999) has been somewhat unclear about whether schema abstraction refers to the problem setting (a problem schema), to the solution strategy (a solution schema), or to both. Problem schemas are clearly critical for improving similarity-based retrieval, since the problem content is all that can possibly be matched between a solved stored problem and an unsolved target problem. Solution schemas likely facilitate transfer by providing a generalized solution strategy to facilitate adapting and applying the retrieved knowledge (Novick \& Holyoak, 1991). Further, a solution schema could potentially be accessed by means other than similarity-based retrieval, such as temporarily heightened accessibility in memory, inclusion in a toolkit of solution strategies, or implied relevance after a didactic presentation. In existing research on analogical retrieval and problem solving, the examples for comparison have always included solutions; accordingly, the resulting generalizations should have included both problem and solution schemas. One goal of the present work is to evaluate whether abstracting just a problem schema could account for the effect of comparison in promoting analogical retrieval.

A further open question is whether schemas must be stored in memory to yield advantages for analogical retrieval. They may not need to be. General processing accounts of memory retrieval have long posited that the quality of the match between a memory probe and a source item stored in memory is as much a function of the encoding of the probe as it is of the source (Tulving \& Thomson, 1973). However, the quality of the encoding of the probe is largely a new topic in research on analogical retrieval. Two recent lines of research suggest that analogical retrieval is sensitive to how memory probes are encoded, and that the effectiveness of an encoding depends on a greater contribution of structural rather than surface content. Lane and Schooler (2004) found that speaking aloud during retrieval strengthened people's biases toward surface-similar memory retrievals. By their account, the retrieval probe emphasized surface features, because these were easier to verbalize than relations, and this dampened analogical retrievals. Gentner, Loewenstein, and Thompson $(2004,2007)$ found that management consultants who compared two analogous negotiation cases - in contrast to those who analyzed the cases separately — were better able to retrieve a further analogous example from their own prior experience (i.e., one learned outside the experimental setting). They also found that schema quality was associated with analogical retrieval. Thus, general accounts of memory retrieval processes, as well as research specifically addressing analogical retrieval, suggest the potential to facilitate analogical problem solving by generating a schematic encoding for use as a retrieval probe.

\section{Overview of the Experiments}

The three experiments that follow examine analogical retrieval by reversing the usual approach of comparing two source stories before attempting a target problem. Instead, we examine whether target comparison-the comparison of two unsolved problems - facilitates analogical retrieval of a single previously read story and the subsequent application of that story's solution to the test problems. Of course, the test problems do not contain solutions. As a result, if comparing two test problems facilitates retrieving a prior analogous story, it cannot be due to having a solution schema. In addition, the compared test problems serve as memory probes, not stored source items; we can therefore test whether having a schema for a memory probe facilitates retrieving an analogous source story. We first tested target comparison in Experiment 1, then we evaluated whether its success depended on comparison (Experiment 2) and upon retrieving the initial story (Experiment 3 ). If comparing problems does indeed facilitate analogical retrieval, we have demonstrated the existence of a technique for promoting analogical transfer at the time of need - a way in which otherwise inert knowledge can in fact be brought to bear (see also Gentner et al., 2004).

\section{EXPERIMENT 1}

Our primary question was whether comparing two unsolved problems encouraged spontaneous retrieval and transfer of a previously read analogue. We used classic materials for evaluating analogical problem solving: Duncker's (1945) Radiation problem, and two analogous problems in different settings (Gick \& Holyoak, 1980, 1983). The extensive experimental tradition (Catrambone, 2002; Catrambone \& Holyoak, 1989; Gick \& Holyoak, 1980, 1983; Grant \& Spivey, 2003; Holyoak \& Koh, 1987; Keane, 1985, 1987; Spencer \& Weisberg, 1986) using these materials provides an advantageous basis for precise investigation of a new question and for grounding current findings. In the baseline condition, participants studied one source-convergence story (we use story to mean a description of both a problem and its solution), then attempted to 
solve a convergence problem. In the source comparison condition, participants compared two source convergence stories set in different domains before attempting the test convergence problem. We expected source comparison to lead to greater transfer of the convergence solution relative to baseline, as in Gick and Holyoak (1983). In the critical target comparison condition, participants read one source convergence story before attempting to jointly solve two convergence problems. We predicted that target comparison participants would generate more convergence solutions than baseline participants. We made no prediction regarding the relative success of source and target comparison. People should generate, in both conditions, a problem schema that promotes analogical retrieval. Source comparison should also lead people to generate solution schemas, which should produce further facilitation if there is more to analogical problem-solving success (with these materials) than achieving a reminding based on matching problem structure.

\section{Method}

Participants. A total of 226 undergraduate students at Binghamton University participated, in partial fulfillment of a course requirement. All relevant consent and human subject approvals were obtained for this and subsequent experiments. Participants were randomly assigned to the baseline $(n=79)$, source comparison $(n=$ $76)$, or target comparison $(n=71)$ conditions.

Materials. The target problem used at test in all conditions was the Radiation problem originally developed by Duncker (1945). The additional cases were analogs based on the convergence principle adapted from Gick and Holyoak (1983). The source story in the study phase of all conditions was The General. In source comparison, Red Adair was presented as a solved story during study. In target comparison, Red Adair was used as a test problem without the last lines explaining the convergence solution.

Procedure. In the baseline condition (1:1), participants were given one source story and one target problem (1:1 indicates the number of initial stories and the subsequent number of test problems). Participants were instructed to read the story The General carefully and to gain sufficient familiarity to be able to retell it in their own words. To promote encoding of the passage, participants were asked to respond in writing to the following question: "What critical insight allowed the problem in the story to be solved?" At test, participants were asked to read the Radiation problem and explain how the problem could be solved. In all conditions, participants did not receive the test packet until they handed in the study packet.

In the source comparison condition (2c:1), participants read and compared two stories: The General, followed by Red Adair. To ensure encoding, they were instructed to prepare to retell the stories and asked to generate the critical insight common to the stories. Participants were encouraged to consider the parallels between the two stories and to complete a matching task in which five story elements of The General had to be matched with elements of Red Adair. ${ }^{1}$ Each element had exactly one appropriate match: General and Red Adair; fortress and burning oil well; army and fire retardant foam; the entire army is needed to capture the fortress and a large amount of foam is needed to extinguish the fire; large groups of men will detonate the mines and no large enough hose is available. The columns were prepared in a jumbled order so that no correctly corresponding elements were directly across from one another. At test, participants attempted the Radiation problem as in the baseline condition.

In the target comparison condition (1:2c), participants studied The General exactly as in baseline. At test, participants were given the Radiation and Red Adair problems with the following comparison instructions:
What approach would you take to solve both of the following problems? After reading the problems carefully, please complete the matching task and then explain your proposed solutions in the space provided. Here's an important hint: The same strategy can be used to solve both problems.

On the following page was a matching task like that used in the source comparison condition. The matches for Radiation, in the same order as those presented above for Red Adair, were doctor; tumor; rays; high intensity must be used; and high intensity will destroy healthy tissue. On the final page, participants were reminded "the same type of solution can be used," and they were asked how to solve these problems.

Scoring. A rater blind to condition scored whether each response solved the Radiation problem in terms of the convergence solution and discussed borderline cases with another rater to produce an agreed-upon scoring. A random sample of 41 responses drawn from all three data sets was presented to one of the authors, who performed a condition-blind coding; the level of agreement was 40 out of $41(98 \%)$. When more than one solution was written, participants were credited with the correct answer if it was among the proposed solutions. As in previous research using the convergence materials, a response was scored as a convergence solution if it captured the principle of a multiplicity of low-intensity rays acting in concert on the tumor. Solutions involving repetition over time rather than in parallel were not counted as convergence solutions. Responses such as "use a lot of low-intensity rays"-for which the application of rays at the same time and to the same area is only implied - were accepted (rejecting them produced a small overall reduction in solution rates that was evenly balanced across conditions).

\section{Results and Discussion}

Target comparison (54\%) dramatically outperformed baseline (15\%; see Table 1$)$ in producing convergence solutions to the Radiation problem $\left[\chi^{2}(1, N=150)=24.72\right.$, $p<.01]$. Source comparison $(41 \%)$ was also reliably more successful than baseline $\left[\chi^{2}(1, N=155)=12.66, p<.01\right]$, replicating previous results using this paradigm (Gick \& Holyoak, 1983). The difference between source and target comparison was not reliable, $\left[\chi^{2}(1, N=147)=2.39, p=\right.$ $.12]$. If the primary hurdle in analogical problem solving is retrieval, and both these conditions foster developing problem schemas that support analogical retrieval, it is reasonable that these two groups performed comparably well.

Despite the well-known difficulty of promoting spontaneous analogical retrieval and problem-solving transfer, comparing two unsolved problems at test yielded substantial gains. Some participants showed explicit evidence of analogical retrieval through direct references to the story The General in their written solutions. For example, one participant in target comparison wrote, "The problems can be solved the same way the general solved his problem."

Table 1

Proportions (and Raw Numbers) of Convergence Solutions by Condition and Experiment

\begin{tabular}{llll}
\hline \multicolumn{1}{c}{ Condition } & Experiment 1 & Experiment 2 & Experiment 3 \\
\hline Target comparison $(1: 2 \mathrm{c})$ & $.54(38 / 71)$ & $.38(27 / 72)$ & $.51(36 / 70)$ \\
Source comparison $(2 \mathrm{c}: 1)$ & $.41(31 / 76)$ & & \\
Baseline $(1: 1)$ & $.15(12 / 79)^{* *}$ & & \\
Separate targets $(1: 2)$ & & $.15(11 / 75)^{* *}$ & \\
Targets only $(0: 2 \mathrm{c})$ & & $.27(20 / 75)$ & $.34(24 / 70)^{*}$ \\
\hline
\end{tabular}

${ }^{*} p<.05,{ }^{* *} p<.01$, for the within-column contrast with the target comparison. 
Successful analogical retrieval in the target comparison condition could not have been based on abstracting the convergence principle, as this was no more likely in this condition than in the baseline condition. Rather, comparing test problems appears to produce a better retrieval probe to access the source story and its convergence solution. The effect of target comparison fits in well with a guiding theme in the problem-solving literature: Problem representation largely determines problem-solving success (Simon, 1978).

In sum, using classic materials, we have demonstrated a robust effect of target comparison. In addition, a further undergraduate sample with an alternative ordering of the convergence materials replicated the effectiveness of target comparison. ${ }^{2}$ In the next two experiments, we attempted to gain further precision in specifying the target comparison advantage for analogical retrieval.

\section{EXPERIMENT 2}

This study tested a variation of the target comparison condition in which participants were not guided to seek a common solution for both problems. This separate targets condition still required participants to generate solutions to two problems, but the specific suggestions to compare and work toward a single solution strategy were removed. If target comparison outperformed separate targets, this would be evidence that the comparison process, not merely the successful solution of more than one problem (Catrambone \& Holyoak, 1989; Loewenstein et al., 1999; Ross \& Kennedy, 1990), is the determining factor. Although it is possible that separate targets might spontaneously compare the two analogous test problems, prior research suggests both that this is unlikely, and that the more thoroughly participants are led to compare cases the greater their transfer is likely to be (Gentner, Loewenstein, \& Thompson, 2003; Kurtz, Miao, \& Gentner, 2001).

We also sought to address whether target comparison specifically depends upon retrieval. People may be better able to solve a difficult problem from first principles if they are given two problems amenable to the same solution strategy. In other words, the effect could be attributable to an improved basis for reasoning or to insight mechanisms, rather than to an improved retrieval probe. Particularly, in light of our instruction to participants that the two problems can be solved in the same way, it is possible that target comparison produced an effect by curtailing exploration of irrelevant aspects of the problem space or-proposed as a useful problem-solving heuristic by Polya (1945) - allowing creation of a more general version of the problem. Our second question, then, is whether target comparison produces better problem solving specifically due to analogical retrieval. We used a targets-only condition $(0: 2 \mathrm{c})$ to assess performance without any prior presentation of a source story, and hence without any example of the convergence solution to retrieve.

\section{Method}

Participants. A total of 222 undergraduate students at Binghamton University participated, in partial fulfillment of a course requirement. Participants were randomly assigned to the target comparison $(n=72)$, separate targets $(n=75)$, or targets-only $(n=75)$ condition.

Materials, Procedure, and Scoring. The target comparison (1:2c) condition exactly followed that in Experiment 1 . The separate targets (1:2) condition differed only in the removal of comparisonencouraging instructions: The initial comparison suggestion and its repetition were excluded. In the targets-only condition $(0: 2 \mathrm{c})$, participants were immediately asked to solve the Radiation and Red Adair problems without prior exposure to the The General. The comparison suggestion was provided once, just before participants were asked to produce their solutions.

\section{Results and Discussion}

Participants in the separate targets condition generated the convergence solution infrequently (15\%; see Table 1$)$ and reliably less often than those in the target comparison condition $(38 \%)\left[\chi^{2}(1, N=147)=9.99, p<.01\right]$. Therefore, an explicit instruction to compare and generate a common solution is critical to the success of target comparison. This rules out explanations in terms of the larger cue set or any interpretation based solely on the presence of two different problems.

With regard to retrieval, the targets-only group (27\%) performed at about the midpoint between the other two groups. The numerically higher rate of transfer in targets only over separate targets was just short of significance $\left[\chi^{2}(1, N=150)=3.29, p=.07\right]$ and a nonsignificant trend suggested less frequent transfer in targets only relative to target comparison $\left[\chi^{2}(1, N=147)=1.98, p=\right.$ .16]. These data suggest that the advantage of target comparison might be attributable to a combination of both improved analogical problem solving and improved strategic problem solving from first principles. However, given the marginal results for targets only, the use of slightly differing instructions, and the potentially idiosyncratic low level of target comparison performance relative to Experiment 1 , we conducted an additional experiment.

\section{EXPERIMENT 3}

We contrasted two conditions that were entirely identical except for presence (target comparison) versus absence (targets only) of the initial convergence story.

\section{Method}

Participants. A total of 140 undergraduate students at Binghamton University participated, in partial fulfillment of a course requirement. Participants were randomly assigned to the target comparison $(n=70)$ or targets-only $(n=70)$ condition.

Materials, Procedure, and Scoring. Target comparison (1:2c) was conducted using the same procedure as that used in Experiments 1 and 2 . The targets-only $(0: 2 \mathrm{c})$ condition differed only in that no solved source analogue was provided. Notably, the comparison suggestion was exactly the same. To gain further insight into the problem-solving process, participants in the target comparison condition were given a posttask questionnaire ${ }^{3}$ in which participants were asked to select one or more of five possible descriptions that matched their experiences. Each choice was a description of a potential strategy (strategy names were not included): (1) figuring out one problem and using it to solve the other problem (piggybacking); (2) seeing what the problems had in common which led to a solution strategy for both (problem encoding); (3) recalling the story 
The General and its applicable solution (analogue retrieval); (4) approaching each problem separately (independent); and (5) solving the problem in some other manner (other). While this measure is obviously indirect and reflects only how participants viewed their own processing, problem solving is a domain in which people can be expected to articulate their experience, as evidenced by the widespread use of verbal protocols. It should also be a conservative measure, because it relies on explicit awareness.

\section{Results and Discussion}

Target comparison once again produced a high rate of convergence solutions $(51 \%)$, significantly more than targets only $(34 \%)\left[\chi^{2}(1, N=140)=4.2, p<.05\right]$ (see Table 1). This difference allows us to infer that target comparison participants were drawing upon the analogous source story, since this was the only difference between the target comparison and targets-only conditions.

Further evidence favoring analogical retrieval was found in the questionnaire results. We contrasted the strategies chosen by participants who did ("solvers") and did not ("nonsolvers") generate convergence solutions (see Table 2). The solvers were significantly more likely to report analogue retrieval (53\% vs. $15 \%)\left[\chi^{2}(1, N=70)=\right.$ $11.25, p<.01]$. Self-report of analogical retrieval of the source story was therefore a good predictor of problemsolving success. The nonsolvers were more likely to report independent processing $(44 \%$ vs. $8 \%)\left[\chi^{2}(1, N=\right.$ $70)=11.72, p<.01]$. Therefore, in those cases where the encouragement to compare problems did not lead participants to adopt a joint solution strategy, a convergence solution was less commonly achieved.

While the convergence solution rate in the targets-only condition was reliably lower than target comparison, performance in that condition did exceed established rates of success on the Radiation problem given on its own (Gick \& Holyoak, 1980) and in our baseline group (1:1), in which the convergence solution was illustrated with a source analogue. This raises the intriguing possibility that target comparison not only promotes analogical problem solving, but can also foster problem solving from first principles (by promoting insight or restricting the hypothesis space). Alternatively, it is possible that targets-only comparison is also a case of retrieval involving a reminder of the individual's personal experience, rather than of a (more accessible) provided source. If so, this is entirely consistent with the primary theme of the paper: that target comparison facilitates analogical retrieval. Further research is under way to explore the role of target comparison without an explicitly provided analogous source.

\section{GENERAL DISCUSSION}

Our central finding is that comparing two unsolved problems promotes analogical retrieval. Comparing examples was critical, since participants in Experiment 2 showed no facilitation when given the test problems without encouragement to solve them jointly. We conclude that comparing test problems facilitates analogical retrieval, not just problem solving in general, since participants in Experiment 3-who did not receive an initial convergence story-were less likely to generate convergence solutions. Among those participants who received the initial story, those who generated convergence solutions were more likely than nonsolvers to say that they drew upon that story. The primary theoretical implication is that comparison-enhanced representation is effective when applied to unsolved problems at the time of test.

Our findings also shed light on the well-known source comparison effect. In addition to providing a replication of Gick and Holyoak (1983) under slight procedural variations, the success of target comparison bears on the theoretical interpretation of source comparison. The retrieval facilitation arising from comparing source examples can now be clearly attributed to the formation of a problem schema (a generalization of a problematic situation), rather than to a solution schema (a generalization of a solution strategy applicable to a range of problems). This follows directly from a similarity-based retrieval framework, since a target problem can match a schema on the problem circumstances, but not on the solution (which is not provided).

Our emphasis has been on how people represent memory probes, rather than, as has been traditionally the case, on how stored items are represented. The present findings show that people do not necessarily have to learn everything correctly the first time in order to achieve successful analogical retrieval and transfer. Rather, if they are able to arrive at a more abstract understanding of their problem, they can access previously experienced structural matches that would otherwise have been inaccessible. Another potential advantage of target comparison is that it ensures and widens the involvement of the generated schema in the retrieval process, because the schema is the probe; that is, the schema participates in every comparison to stored cases, in contrast to a stored schema that, presumably, is only involved once. As an applied technique, target comparison offers the promise that ordinary experience need not be analogically inert.

Table 2 Post-Questionnaire Responses From the Target Comparison Condition of Experiment 3

\begin{tabular}{lcccrr}
\hline & \multicolumn{4}{c}{ Problem-Solving Strategy } \\
\cline { 2 - 5 } & Piggybacking & $\begin{array}{l}\text { Problem } \\
\text { Encoding }\end{array}$ & $\begin{array}{l}\text { Analogue } \\
\text { Retrieval }\end{array}$ & Independent & Other \\
\hline Convergence solution $(n=36)$ & $31 \%$ & $56 \%$ & $53 \%{ }^{* *}$ & $8 \%$ & $3 \%$ \\
No-convergence solution $(n=34)$ & $15 \%$ & $35 \%$ & $15 \%$ & $44 \%{ }^{* *}$ & $18 \%$ \\
\hline $\begin{array}{l}\text { Note-Participants could choose as many strategies as they wished, hence the percentages sum to } \\
\text { greater than } 100 . \quad{ }^{* *} p<.01 \text { for the column contrast. }\end{array}$
\end{tabular}


The target comparison advantage suggests that having a second problem to compare with a target problem - even without knowing a solution to the additional problem — can improve the encoding of memory probes. Naturally occurring situations amenable to target comparison require the availability of multiple problems with a common solution, as well as some reason to treat such problems jointly. We speculate that explicit information about both problems sharing a solution will not turn out to be critical, because it is just one of many ways of fostering deep comparison (such as writing a joint explanation or listing deep and interesting commonalities). Problems can also be brought into comparison through spatial or temporal juxtaposition, perhaps due to social interaction. People may also retrieve a comparison case from past experience. Such remindings are likely to be surface-based, but remindings initially found through surface similarity can yield useful structural insights (Gentner, 1989; Ross \& Kennedy, 1990). Further, lower order structural content can lead to similarity-based access as effectively as surface content (Catrambone, 2002). Importantly, target comparison does not require the availability of a perfect analogue - it may produce benefits based on partial structural consistency, as long as there is a sufficient basis for the mechanisms of highlighting, rerepresentation, or abstraction. From a pedagogical standpoint, the issue may be one of teaching a metacognitive strategy of seeking out additional problems that act not only as analogies to support potential solutions, but also to refine the encoding and interpretation of the focal problem.

Target comparison also offers a way to situate learning in the context of active problem solving. Successful retrieval of an analogue encourages schema abstraction in its own right (Novick \& Holyoak, 1991; Ross \& Kennedy, 1990). Thus, target comparison, by enabling the productive retrieval of analogs that were routinely (i.e., individually) encoded at the time of study, may gradually generate conceptual change from novice to expert representations of a domain (Carey, 1995; Gentner \& Loewenstein, 2002). One area of application is in teaching adults, who often come to the classroom less for content knowledge than for analytical frameworks that make sense of their years of work experience. For example, the Gentner et al. $(2004,2007)$ studies discussed in the introduction showed that management consultants learning to negotiate were better able to retrieve analogous examples from their own memories when they were encouraged to compare rather than separately analyze examples. Rather than ignoring their prior knowledge or considering it just a source of bad habits, we can now suggest a way for it to be productively mined.

Three directions stand out for further study. The first is evaluating target comparison under conditions of temporally remote transfer. The traditional source comparison advantage tends to fade with temporal distance or a dissimilar task setting (Spencer \& Weisberg, 1986), except when a more substantial intervention with more than two examples is used (Catrambone \& Holyoak, 1989; Chen \& Klahr, 1999). The benefit from target comparison may be less likely to diminish. The second direction to explore is the suggestion in our data that target comparison promotes not only memory-based problem solving, but also problem solving from scratch without a provided analogue. Finally, we predict that target comparison can improve problem encoding without explicitly informing participants that both problems can be solved in the same manner. We plan to explore alternate techniques for inviting robust comparison (e.g., joint explanation or commonality listing tasks).

\section{AUTHOR NOTE}

We are grateful for many insightful discussions on these issues with Dedre Gentner. We greatly appreciate the hard work and thoughtful contributions of Jessica Federman, Aliza Nelson, Olga Boukrina, Janeen O'Connor, and the members of the Learning and Representation in Cognition (LaRC) Lab at Binghamton University. We also thank Bobbie Spellman, Christian Schunn, and anonymous reviewers for helpful comments. Correspondence concerning this article should be addressed to K. J. Kurtz, Department of Psychology, Binghamton University, P.O. Box 6000, Binghamton, NY 13902 (e-mail: kkurtz@binghamton.edu).

\section{REFERENCES}

Barnett, S. M., \& Ceci, S. J. (2002). When and where do we apply what we learn? A taxonomy for far transfer. Psychological Bulletin, 128, 612-637.

CAREY, S. (1995). Continuity and discontinuity in cognitive development. In E. E. Smith \& D. N. Osherson (Eds.), Thinking: An invitation to cognitive science (2nd ed., Vol. 3, pp. 101-129). Cambridge, MA: MIT Press.

Catrambone, R. (2002). The effects of surface and structural feature matches on the access of story analogs. Journal of Experimental Psychology: Learning, Memory, \& Cognition, 28, 318-334.

Catrambone, R., \& Holyoak, K. J. (1989). Overcoming contextual limitations on problem-solving transfer. Journal of Experimental Psychology: Learning, Memory, \& Cognition, 15, 1147-1156.

CHEN, Z., \& KlAHR, D. (1999). All other things being equal: Acquisition and transfer of the Control of Variables Strategy. Child Development, 70, 1098-1120.

Clement, C. A., \& Gentner, D. (1991). Systematicity as a selection constraint in analogical mapping. Cognitive Science, 15, 89-132.

Clement, C. A., Mawby, R., \& Giles, D. E. (1994). The effects of manifest relational similarity on analog retrieval. Journal of Memory \& Language, 33, 396-420.

Cummins Dellarosa, D. (1992). Role of analogical reasoning in the induction of problem categories. Journal of Experimental Psychology: Learning, Memory, \& Cognition, 18, 1103-1124.

DunBar, K. (2001). The analogical paradox: Why analogy is so easy in naturalistic settings yet so difficult in the psychology laboratory. In D. Gentner, K. J. Holyoak, \& B. Kokinov (Eds.), The analogical mind: Perspectives from cognitive science (pp. 313-334). Cambridge, MA: MIT Press.

Duncker, K. (1945). On problem-solving (L. S. Lees, Trans.). Psychological Monographs, 58, No. 270.

Forbus, K. D., Gentner, D., \& LAW, K. (1995). MAC/FAC: A model of similarity-based retrieval. Cognitive Science, 19, 141-205.

Gentner, D. (1983). Structure-mapping: A theoretical framework for analogy. Cognitive Science, 7, 155-170.

Gentner, D. (1989). The mechanisms of analogical learning. In S. Vosniadou \& A. Ortony (Eds.), Similarity and analogical reasoning (pp. 199-241). New York: Cambridge University Press.

GeNTNER, D., \& LoEWENSTeIN, J. (2002). Learning: Analogical reasoning. In J. W. Guthrie (Ed.), Encyclopedia of education (2nd ed.). New York: Macmillan.

Gentner, D., Loewenstein, J., \& Thompson, L. (2003). Learning and transfer: A general role for analogical encoding. Journal of Educational Psychology, 95, 393-408.

Gentner, D., Loewenstein, J., \& Thompson, L. (2004). Analogical encoding: Facilitating knowledge transfer and integration. In K. Forbus, D. Gentner, \& T. Regier (Eds.), Proceedings of the Twenty-Sixth Annual Conference of the Cognitive Science Society (pp. 452-457). Mahwah, NJ: Erlbaum.

Gentner, D., Loewenstein, J., \& Thompson, L. (2007). Analogical 
encoding supports retrieval of prior analogs as well as transfer to future analogs. Manuscript in preparation, Northwestern University.

Gentner, D., \& Medina, J. (1998). Similarity and the development of rules. Cognition, 65, 263-297.

Gentner, D., \& Rattermann, M. J. (1991). Language and the career of similarity. In S. A. Gelman \& J. P. Byrnes (Eds.), Perspectives on language and thought: Interrelations in development (pp. 225-277). Cambridge: Cambridge University Press.

Gentner, D., Rattermann, M. J., \& Forbus, K. D. (1993). The roles of similarity in transfer: Separating retrievability from inferential soundness. Cognitive Psychology, 25, 524-575.

Gick, M. L., \& HOLYOAK, K. J. (1980). Analogical problem solving. Cognitive Psychology, 12, 306-355.

Gick, M. L., \& HolyOAK, K. J. (1983). Schema induction and analogical transfer. Cognitive Psychology, 15, 1-38.

Goldstone, R. L., \& Sakamoto, Y. (2003). The transfer of abstract principles governing complex adaptive systems. Cognitive Psychology, 46, 414-466.

Grant, E. R., \& Spivey, M. J. (2003). Eye movements and problem solving: Guiding attention guides thought. Psychological Science, 14, $462-466$

HoLYOAK, K. J., \& KoH, K. (1987). Surface and structural similarity in analogical transfer. Memory \& Cognition, 15, 332-340.

Hummel, J. E., \& HolyoAK, K. J. (1997). Distributed representations of structure: A theory of analogical access and mapping. Psychological Review, 104, 427-466.

Keane, M. T. (1985). On drawing analogies when solving problems: A theory and test of solution generation in an analogical problemsolving task. British Journal of Psychology, 76, 449-458.

KURTZ, K. J. (2005). Re-representation in comparison: Building an empirical case. Journal of Experimental \& Theoretical Artificial Intelligence, 17, 447-459.

Kurtz, K. J., Miao, C., \& Gentner, D. (2001). Learning by analogical bootstrapping. Journal of the Learning Sciences, 10, 417-446.

LANe, S. M., \& Schooler, J. W. (2004). Skimming the surface: Verbal overshadowing of analogical retrieval. Psychological Science, 15, 715-719.

Loewenstein, J., Thompson, L., \& Gentner, D. (1999). Analogical encoding facilitates knowledge transfer in negotiation. Psychonomic Bulletin \& Review, 6, 586-597.

Medin, D. L., \& Ross, B. H. (1989). The specific character of abstract thought: Categorization, problem solving, and induction. In R. J. Sternberg (Ed.), Advances in the psychology of human intelligence (Vol. 5, pp. 189-223). Hillsdale, NJ: Erlbaum.

Novick, L. R. (1988). Analogical transfer, problem similarity, and expertise. Journal of Experimental Psychology: Learning, Memory, \& Cognition, 14, 510-520.

Novick, L. R., \& HoLYOAK, K. J. (1991). Mathematical problem solving by analogy. Journal of Experimental Psychology: Learning, Memory, \& Cognition, 17, 398-415.
Polya, G. (1945). How to solve it: A new aspect of mathematical method. Princeton, NJ: Princeton University Press.

ReEves, L. M., \& Weisberg, R. W. (1994). The role of content and abstract information in analogical transfer. Psychological Bulletin, 115, 381-400.

Ross, B. H. (1989). Distinguishing types of superficial similarities: Different effects on the access and use of earlier problems. Journal of Experimental Psychology: Learning, Memory, \& Cognition, 15, 456-468.

Ross, B. H., \& Kennedy, P. T. (1990). Generalizing from the use of earlier examples in problem solving. Journal of Experimental Psychology: Learning, Memory, \& Cognition, 16, 42-55.

SimON, H. A. (1978). Information-processing theory of human problem solving. In W. K. Estes (Ed.), Human information processing (Handbook of Learning and Cognitive Processes, Vol. 5, pp. 271-295). Hillsdale, NJ: Erlbaum.

SPENCER, R. M., \& WeISBERG, R. W. (1986). Context-dependent effects on analogical transfer. Memory \& Cognition, 14, 442-449.

Tulving, E., \& Thomson, D. M. (1973). Encoding specificity and retrieval processes in episodic memory. Psychological Review, 80, 352-373.

\section{NOTES}

1. This procedure has been found to be effective in previous research (Kurtz, Miao, \& Gentner, 2001; Loewenstein et al., 1999), and represents a more minimal manipulation than Gick and Holyoak's (1983) procedure that employs written summaries, ratings of comprehensibility, a similarity listing task, and a similarity rating.

2. In Experiment 1, we held constant the use of the story The General as a source and Radiation as the target problem, and varied our use of Red Adair as either a source story or target problem in order to maintain consistency between the source comparison and target comparison groups. In an additional pilot study, 86 new participants were randomly assigned to one of two versions of the target comparison condition: One received the materials ordered in the same way, and the other saw a reversed version with Red Adair as the source story and The General as a target problem that was compared with the Radiation problem. Participants performed similarly well on the replication $(51 \%)$ and reversed $(40 \%)$ versions of the target comparison condition $\left[\chi^{2}(1, N=86)=\right.$ $1.17, p=.28]$, and at levels comparable to the target comparison and source comparison conditions in Experiment 1. These additional data serve as a replication of performance in the target comparison condition and also extend the finding by showing similar performance with a different comparison problem at test.

3. A subset of 30 participants in the targets-only group was also given the posttask questionnaire, but because this $n$ is small and this group had a different rate of solving from the overall targets-only group, we could derive no conclusions from these data. 


\section{APPENDIX}

Experimental Materials

\section{The General: Problem}

A small country was ruled from a strong fortress by a dictator. The fortress was situated in the middle of the country, surrounded by farms and villages. Many roads led to the fortress through the countryside. A rebel general vowed to capture the fortress. The general knew that an attack by his entire army would capture the fortress. He gathered his army at the head of one of the roads, ready to launch a full-scale direct attack. However, the general then learned that the dictator had planted mines on each of the roads. The mines were set so that small bodies of men could pass over them safely, since the dictator needed to move his own troops and workers to and from the fortress. However, any large force would detonate the mines. Not only would this blow up the road, but it would also destroy many neighboring villages. It seemed impossible to capture the fortress.

\section{The General: Solution}

However, the general devised a simple plan. He divided his army into small groups and dispatched each group to the head of a different road. When all was ready, he gave the signal and each group marched down a different road. Each group continued down its road to the fortress, so that the entire army arrived together at the fortress at the same time. In this way, the general captured the fortress and overthrew the dictator.

\section{Radiation: Problem}

Suppose you are a doctor faced with a patient who has a malignant tumor in his stomach. To operate on the patient is impossible, but unless the tumor is destroyed, the patient will die. A kind of ray, at a sufficiently high intensity, can destroy the tumor. Unfortunately, at this intensity the healthy tissue that the rays pass through on the way to the tumor will also be destroyed. At lower intensities the rays are harmless to healthy tissue, but will not affect the tumor. How can the rays be used to destroy the tumor without injuring the healthy tissue?

\section{Red Adair: Problem}

An oil well in Saudi Arabia exploded and caught fire. The result was a blazing inferno that consumed an enormous quantity of oil each day. After initial efforts to extinguish it failed, famed firefighter Red Adair was called in. Red knew that the fire could be put out if a huge amount of fire retardant foam could be dumped on the base of the well. There was enough foam available at the site to do the job. However, there was no hose large enough to put all the foam on the fire fast enough. The small hoses that were available could not shoot the foam quickly enough to do any good. It looked like there would have to be a costly delay before a serious attempt could be made. [How can the fire be put out immediately?]

\section{Red Adair: Solution}

However, Red Adair knew just what to do. He stationed men in a circle all around the fire, with all of the available small hoses. When everyone was ready, all the hoses were opened up and the foam was directed at the fire from all directions. In this way a huge amount of foam quickly struck the source of the fire. The blaze was extinguished, and the Saudis were satisfied that Red had earned his three-million-dollar fee. 